\title{
Das HERON-Projekt - Middleware für Image Retrieval
}

\section{The HERON-Project - Middleware for Image Retrieval}

\author{
Werner Kießling, Wolf-Tilo Balke, Thomas Birke, Katharina Urch, Matthias Wagner, Universität Augsburg
}

\begin{abstract}
Das HERON-Projekt untersucht die Auswirkungen multimedialer Datenbanktechnologie auf weite Anwendungsbereiche in den Geisteswissenschaften am Beispiel der Heraldik. Besonderer Wert wird dabei sowohl auf fachspezifische Erschließung und intuitive Zugänge zu Bildmaterialien durch den Einsatz einer inhaltsbasierten Suche, als auch auf effiziente Internetanbindung gelegt. Während für die Erschließung des Bildmaterials effektive Segmentierungsalgorithmen zur Vorbearbeitung entwickelt werden, legt die Architektur des prototypischen HERON-Recherchesystems ihren Schwerpunkt auf den Einsatz innovativer Middleware zur effizienten Integration und Auslieferung der multimedialen Anfrageergebnisse.
\end{abstract}

The HERON-project investigates the impact of multimedia database technology on applications in the humanities using heraldry as a sample application. Besides digitizational tasks the main focus is on intuitive retrieval of image material by content-based queries and efficient Internet services. For the preparation of image material effective algorithms for image segmentation have been developed. The architecture of the prototypical HERONsystem aims at implementing innovative middleware for efficient integration and delivery of multimedia result sets.

\section{Einführung}

Einen neuen und sich rasch entwickelnden Zweig der Datenbankforschung und Datenbankentwicklung bilden Multimediadatenbanksysteme, die multimediale Daten wie Bilder, Volltexte, Audio- und Videosequenzen in Datenbanksysteme integrieren $[3 ; 10]$. Eine spezielle Ausprägung dieser Systeme sind Bilddatenbanksysteme [6;8]. Viele dieser Systeme bieten lediglich den Zugriff auf Bildmaterial über verbale Annotationen oder komplexe Grammatiken zur Bildbeschreibung [9]. Im Gegensatz dazu erlauben moderne Systeme nicht nur einen navigierenden Zugriff auf das gespeicherte Bildmaterial, sondern auch eine bildinhaltliche Recherche $[1 ; 2]$. Hierzu werden aus den Bildern graphische Merkmale (sog. Features) extrahiert, welche z. B. die Farbe, Oberflächenstrukturen und die im Bild auftretenden Formen charakterisieren. Durch Vergleich der Features von gespeicherten Bildern und der Anfrage, die z. B. in Form eines Beispielbildes oder auch einer Skizze vorliegen kann, wird eine geordnete Liste derjenigen Bilder bestimmt, die der Anfrage möglichst nahe kommen, also bezüglich der Features ähnlich sind.

Das HERON-Projekt wird interdisziplinär vom Lehrstuhl für Datenbanken und Informationssysteme und der Universitätsbibliothek der Universität Augsburg getragen [7]. Als Anwendungsbereich wurde die kunsthistorische Forschung gewählt, die besonders stark durch das Vorhandensein von Bildmaterial geprägt ist. Im ersten Stadium des Projekts wurde dabei die Heraldik, also Wappenkunde betrachtet. Sie verfügt über eine extrem standardisierte Bildsprache, die sich durch den Gebrauch stark stilisierter Bildformen auszeichnet. Zudem besitzt die Heraldik eine streng formalisierte verbale Beschreibungssprache (sog. Blasonierungen) dieser Formen und ihrer Bedeutung. Bildbestandteile sind also nicht nur deutlich $\mathrm{zu}$ unterscheiden, sondern auch immer eindeutig einer Klasse von Abbildungsformen zuzuordnen.

Das prototypische HERON-System wurde als DreiSchichten-Architektur konzipiert und soll in das World Wide Web integriert werden. Hierbei wird 
spezifischen Forderungen der Anwender Rechnung getragen und insbesondere die Art und Qualitätsstufe der vom Client angeforderten Bildformate berücksichtigt. Sowohl die Datenspeicherung als auch die Datenübertragung wird anhand dieser Parameter optimiert. Auch für die effiziente Kombination und Gewichtung einzelner bewerteter Ergebnislisten miteinander wurde bereits ein Algorithmus entwickelt. Parallel zum Aufbau des Datenbanksystems wurde ein Standardwerk, welches als heraldische Datenbasis nicht nur qualitativ hochwertiges Bildmaterial, sondern auch die vollständigen verbalen Beschreibungen enthält, fachlich fundiert erschlossen und digitalisiert [11]. Anhand dieser historischen Wappendarstellungen wurden verschiedene bildinhaltliche Recherche-Operatoren evaluiert und Algorithmen zur Segmentierung der in den Bildern enthaltenen Formen entwickelt.

Neben dem umfangreichen Bildmaterial stellen die historischen Originalquellen auch fachliche Textinformation, z. B. eine genaue Bildbeschreibung oder genealogische Information, zur Verfügung. Solche MetaDaten haben einen großen Wert gerade auch für Bildrecherchesysteme [9]. Sie bieten nicht nur allgemeine Information bezüglich der recherchierten Ergebnisbilder, sondern befähigen Experten dazu, schnell Informationen über bekanntes Bildmaterial $\mathrm{zu}$ erhalten oder eigene Vermutungen verifizieren $\mathrm{zu}$ können. Unter einer vollen Erschließung versteht man also nicht nur die Digitalisierung von Wappendarstellungen, sondern auch die fachlich fundierte redaktionelle Aufarbeitung und OCR-Behandlung von Texten, die im Bezug zum Bildmaterial stehen. Eine genauere Darstellung der Vorgehensweise zur Digitalisierung im HERON-Projekt unter Berücksichtigung der besonderen geisteswissenschaftlichen Bezüge und Problemstellungen gibt [11].

Im Rahmen eines computergestützten kunsthistorischen Arbeitsplatzes werden darüber hinaus die Erfahrungen mit der bildinhaltlichen Recherche in Bilddokumenten für digitale Bibliothekssysteme erschlossen. Das HERON-Systems dient dabei als kunsthistorisches Internet-Portal. Im Blickpunkt steht des Weiteren die Ausdehnung auf andere Anwendungsbereiche weit über die kunsthistorische Forschung hinaus und die Evaluierung der Anwendbarkeit in allgemeinen kommerziellen Fotoarchiven.

\section{Bildinhaltliche Recherche}

Features für die bildinhaltliche Suche lassen sich aufgliedern in Farb-, Textur- oder Formfeatures. In allen Features gibt es dabei die Möglichkeit, die Position auf dem Originalbild mit einzubeziehen. Die größte Herausforderung in der Aufbereitung eines Anwendungsbereichs ist die spezifische Zuordnung der bildinhaltlichen Merkmale zu ihrer semantischen Aussa- ge im Anwendungsgebiet. Speziell auf dem Gebiet der Heraldik lässt sich die Nutzung der Features leicht zuordnen [7]:

- Farbfeatures sind in ihrer Anwendbarkeit beschränkt. Die Heraldik verwendet nur wenige Farben, welche häufig in schwarzweißen Vorlagen durch Schraffierungen codiert sind.

- Texturfeatures können zwar nicht auf Schraffierungen, aber auf spezielle Muster, wie etwa Pelzwer$\mathrm{ke}$, angewendet werden.

- Formfeatures können den Umriss der dargestellten Figuren beschreiben.

- Die Einbeziehung der Position ist wichtig, um z. B. die Anzahl der dargestellten Gegenstände zu bestimmen.

\subsection{Nutzung von Farbfeatures}

Kommerzielle bildinhaltliche Recherchesysteme verwenden fast immer Farbfeatures zur bildinhaltlichen Suche. Da der Anwendung in der Heraldik lediglich monochrome Wappenbilder als Datenbasis zugrunde liegen, ist die Erzeugung von Farbhistogrammen durch visuelle Recherchesysteme nicht direkt möglich. Mit Methoden der digitalen Bildverarbeitung können jedoch Farbhistogramme und Farbskizzen einfarbiger Wappenbilder berechnet werden [12]. Diese können als Grundlage für eine farbbasierte bildinhaltliche Suche dienen. Zum Beispiel wird in Bild 1 die diagonale Schraffierung a), welche die heraldische Farbe Grün codiert, grün (hier in der S/WDarstellung hellgrau) eingefärbt, weiße Teile des Schildes bleiben weiß und graue Felder im Texturbild b) konnten nicht eindeutig zugeordnet werden, bleiben also unbestimmt. Einzelne farblich falsch zugeordnete Punkte treten i.a. zwar im Texturbild auf, spielen allerdings für die Weiterverarbeitung keine Rolle. Dieses Verfahren ist aufgrund der groben Auflösung des Texturbildes leider nicht ausreichend, um Formen klar segmentieren zu können, dennoch kann es dazu genutzt werden, aus den verschieden schraffierten Flächen von Wappen Farbverteilungshistogramme zu erzeugen, wie etwa in Bild 1c). Das Texturbild seinerseits bietet sich gerade durch seine grobe Auflösung zur vergleichenden Anfrage mit ei-
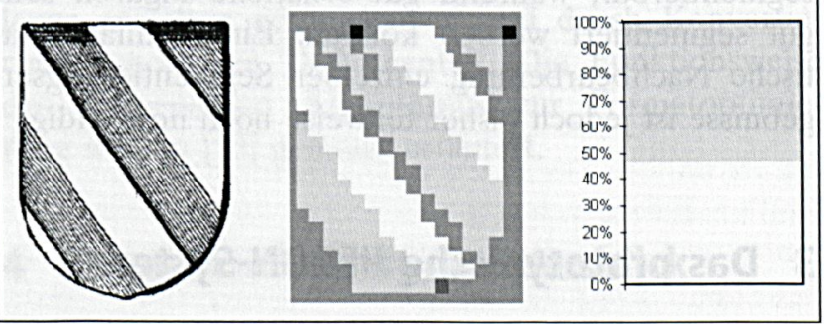

Bild 1: Ein Wappen a), sein Texturbild b) und das erzeugte Farbhistogramm c). (Bemerkung: In dieser S/W-Darstellung ist grün durch hellgrau ersetzt). 


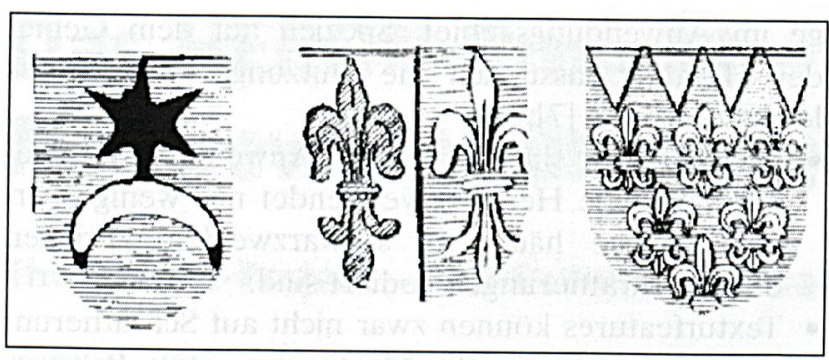

Bild 2: Verschiedene Wappen und ihre automatisch segmentierten Objekte (dunkel umrandet).

genen Bildern oder handgezeichneten Skizzen an (Query by visual example / Query by Sketch).

\subsection{Automatische Segmentierung}

Ein zweiter noch wesentlicherer Schritt in der Vorverarbeitung ist die automatische Segmentierung von auftretenden Formen. Ziel für die Anwendung in der $\mathrm{He}-$ raldik ist die Freilegung der abgebildeten Figuren, die im Regelfall auf einem schraffierten Hintergrund abgebildet sind und so nicht direkt segmentiert werden können. Solche Schraffierungen stellen für gängige Segmentierungsverfahren ein erhebliches Problem dar. Diese beruhen häufig auf der Annahme, dass zu segmentierende Flächen in einer (fast) einheitlichen Farbe vorliegen und ihre Umrandung einen deutlichen Farbunterschied zum Hintergrund darstellt. Leider gehen Schraffierungen fast immer direkt in die Objektkonturen über und machen so auch den Einsatz von z. B. line-tracking-Verfahren unmöglich. Entwickelt wurde hier ein Erfolg versprechendes Verfahren, welches zuerst einen Teil der Schraffierung in einer spezifischen Richtung entfernt, um dann die abgebildeten Formen auf einem weißem Hintergrund zum Beispiel mittels morphologischer Verfahren auszeichnen zu können [12]. Bei der Entfernung von Schraffierungen beschränkt es sich dabei auf die Entfernung der Hintergrundschraffierung, da sonst die Gefahr der übermäßigen Beschädigung von Figuren und deren Umrissen besteht.

Die Qualität der Ergebnisse hängt also einerseits vom Schraffierungsentfernungsverfahren, andererseits aber auch stark von der Beschaffenheit der Vorlage ab. Generell sind fragmentierte Objekte auf bruchstückhaften Schraffierungen praktisch kaum segmentierbar, während gut erhaltene Figuren sehr gut segmentiert werden können. Eine semiautomatische Nachbearbeitung einzelner Segmentierungsergebnisse ist jedoch bisher teilweise noch notwendig.

\section{Das prototypische HERON-System}

Zum Aufbau eines prototypischen Systems im Rahmen des kunsthistorischen Arbeitsplatzes wurde IBM DB2 als unterliegende Datenbank gewählt und die
DB2 Relational Image und Text Extender für die Anwendung in der bildinhaltlichen und Volltext-Recherche [2]. Einen zusätzlichen Wert sowohl für den Fachwissenschaftler als auch für den interessierten Laien bietet das HERON-System mit seinem integrierten Bildthesaurus, der die Suche nach bestimmten Bildelementen durch deren exemplarische Darstellung erleichtert. Durch die begleitenden Bildbeispiele wird der hierarchisch geordnete Index von Sachbegriffen, die mit den bildinhaltserschließenden Texten verknüpft sind, für Laien, welche die genaue Fachterminologie nicht beherrschen, aber auch für fremdsprachliche Benutzer anwendbar. Die Integration eines englischen und eines französischen Fachthesaurus ist als zusätzlicher Schritt geplant.

Eine wesentliche Designentscheidung war der Aufbau des HERON-Systems durch ComponentwareTechnologie aus kommerziellen Produkten und Eigenentwicklungen. Für HERON ist eine MehrSchichten-Architektur vorgesehen (vgl. Bild 3). Zwischen der Server-Seite, welche die kommerziellen Datenbank- und Recherchekomponenten enthält, und der Client-Seite, die eine grafische Oberfläche zur Komposition komplexer Anfragen zur Verfügung stellt, wurde besonderer Wert auf eine effiziente Middleware gelegt. Diese Middleware dient zur Verteilung komplexer Anfragen, die sowohl bildinhaltliche, als auch Volltextanteile enthalten können, zur Erzeugung des Gesamtergebnisses und schließlich zur Auslieferung des Bildmaterials nach anwendungsspezifischen Gesichtspunkten. Die Implementierung erfolgte in reinem Java-Code, um ein Maximum an Plattformunabhängigkeit gewährleisten zu können.

\subsection{Die Komponenten des HERON-Systems}

Das HERON-System besteht aus vier hauptsächlichen Komponenten:

1. Digitalisierung und Erschließung

2. Query Engine

3. Combining Engine

4. Multimedia Delivery Engine

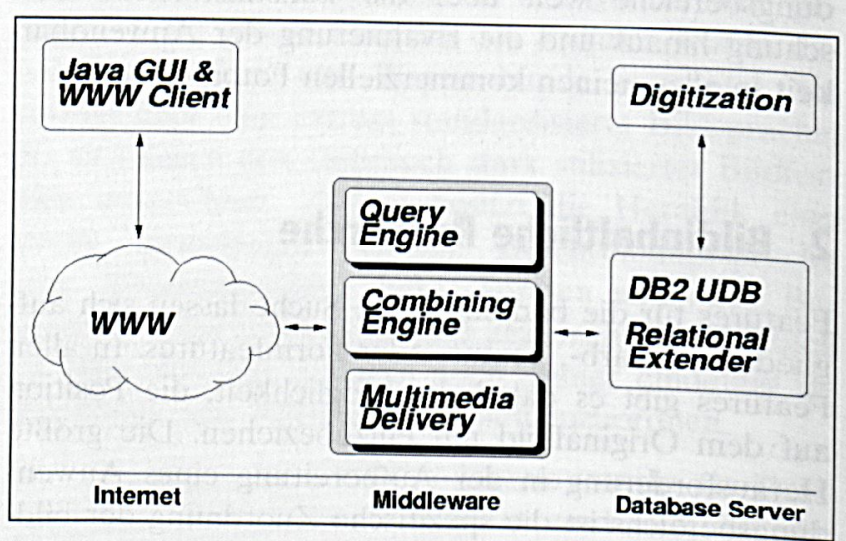

Bild 3: Schichtenarchitektur des HERON-Systems. 


\subsection{Komponenten der HERON-Middleware}

Die Query Engine nimmt komplexe Anfragen des Benutzers auf, unterteilt sie in ihre atomaren Anteile und gibt diese an die einzelnen unterliegenden Systeme weiter. Dieser Teil des Systems ist insbesondere unverzichtbar, wenn eine Ausweitung auf heterogene Datenquellen oder sogar auf heterogene Rechercheund Datenbanksysteme erfolgt. Die Query Engine trägt keine Recherchefunktion, sondern ist lediglich dazu vorgesehen, die Offenheit des Systems gegenüber anderen Komponenten zu gewährleisten.

Die Combining Engine ist konzeptionell das Gegenstück zur Query Engine. Sie dient zur Vorbereitung der Ausgabe von Anfrageergebnissen. In der Combining Engine werden sämtliche einzelnen Anfrageergebnisse aufgenommen und mit Hilfe eines neuartigen Algorithmus nach einer beliebig vorgegebenen gewichteten Kombinationsfunktion zu einem komplexen Anfrageergebnis zusammengefasst [5]. Das allgemeine Anwenderprofil zeigt hier, dass Anwender für komplexe Anfragen im Allgemeinen nicht an der vollständigen Datenbasis als Ergebnis interessiert sind, sondern nur an einem geringen Teil, der die besten Objekte bezüglich der Anfrage innerhalb der gesamten Datenmenge beinhaltet [4].

Die Grundidee für einen effizienten Kombinationsalgorithmus ist, verschiedene atomare Ergebnislisten gewichtet zu einem Gesamtergebnis zusammenzufassen, ohne dabei die irrelevanten Teile jeder einzelnen Liste aus der Datenbank abfragen zu müssen. Ein solcher vollständiger Aufbau der Ergebnisliste könnte nämlich nur durch einen ineffizienten Scan über die gesamte Datenbasis generiert werden. Der entwickelte Algorithmus „Quick-Combine“ [5] hat hierbei sowohl in praktischen Fallstudien, als auch in ausführlichen Tests auf synthetischen Daten seine Überlegenheit gegenüber bestehenden Ansätzen gezeigt. Quick-Combine weist dabei im Durchschnitt Performanzsteigerungen mit Faktoren der Größenordnung 30 auf. Aufgrund seiner Ablaufsteuerung passt sich der Algorithmus ideal jeder Datenverteilung an und skaliert mit wachsender Datenbankgröße. QuickCombine befindet sich derzeit in der Patentierung unterstützt durch die DFG-Ideenwerkstatt.

Die Multimedia Delivery Engine beschäftigt sich mit zwei verschiedenen Aspekten der HERON-Datenbank, nämlich der Haltung und der Auslieferung von Bildmaterial. Der generelle Ansatz ist jedoch auf verschiedene Arten von Multimediadokumenten übertragbar. Multimediadokumente liegen in Digitalen Bibliotheken oftmals in unterschiedlichen Formaten vor, die zueinander in funktionalen Abhängigkeiten stehen, $d . h$. durch geeignete Formatkonvertierungen ineinander überführt werden können. Bei der Auslieferung von Multimediadokumenten muss insbesondere spezifischen Forderungen von Seiten der Anwender aus unterschiedlichsten Benutzerkreisen

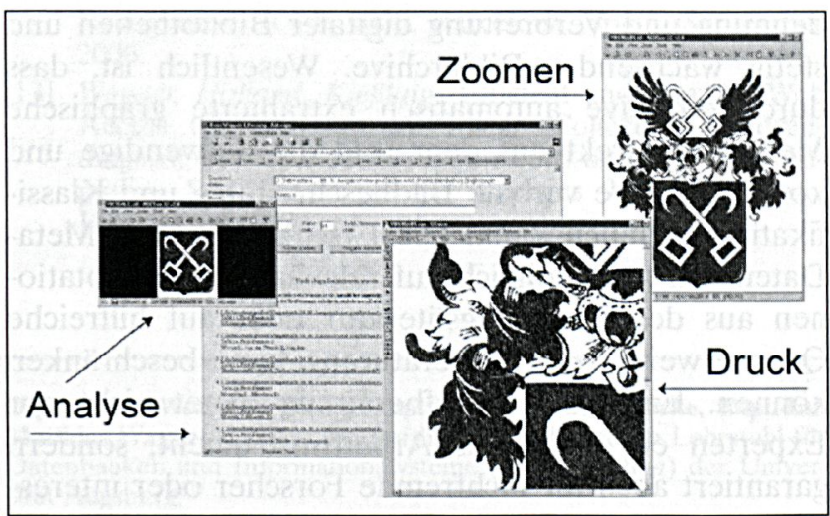

Bild 4: Verschiedene Dateiformate und Qualitätsstufen werden für verschiedene Arbeitsschritte benötigt.

Rechnung getragen werden [13]. Eine zentrale Aufgabe dabei ist die Verfügbarkeit von Bildmaterial in unterschiedlichen Qualitätsstufen und Bildformaten, wie sie bei typischen mehrstufigen Arbeiten benötigt werden (s. Bild 4). Während man beispielsweise für die erste Analyse einer Treffermenge nur Thumbnails benötigt, sind für die weiteren Arbeitsschritte wie Zoomen und Drucken qualitativ hochwertigere Formate in höheren Auflösungen nötig. Existierende kommerzielle Datenbanksysteme wie IBM DB2 sehen bereits die Möglichkeit zur Auslieferung von Bildmaterial in diversen Formaten und Qualitätsstufen vor. Doch ist in diesen Systemen primär nur die Speicherung eines Referenzformats vorgesehen, aus welchem alle anderen Formate für die Auslieferung konvertiert werden müssen.

Abhilfe schafft hier die Speicherung häufig angefragter Formate in der Datenbank. Mit sich veränderndem Anfrageprofil müssen auch die in der Datenbank abgelegten Formate geändert werde. Die Auswahl von Speicherformaten gestaltet sich allerdings problematisch:

- Einerseits werden zum Preis von hohen Speicherkosten Dokumente in der Datenbank gespeichert, die selten angefragt werden und zudem mit geringem Kostenaufwand aus äquivalenten Dokumenten in anderen Formaten gewonnen werden können.

- Andererseits werden häufig angefragte Dokumente nicht gespeichert, obwohl ihre Online-Konvertierung sehr teuer ist.

Ziel einer Format-Optimierung zum Austausch von Multimediadokumenten ist die kostenoptimale $\mathrm{Ba}$ lance zwischen gespeicherten und durch Konvertierung generierten Dokumenten. Die Funktionsweise des verwendeten Algorithmus zur Formatoptimierung wird in [13] genauer erläutert.

\section{Zusammenfassung und Ausblick}

Die Nutzbarkeit von bildinhaltlichen Recherchesystemen zur effizienten Handhabung großer Bildbestände ist eine zentrale Fragestellung im Zuge der Aus- 
dehnung und Verbreitung digitaler Bibliotheken und stetig wachsender Bildarchive. Wesentlich ist, dass durch effektive automatisch extrahierte graphische Merkmale direkt aus dem Bild die aufwendige und kostenintensive verbale Bildbeschreibung und Klassifikation entfallen kann. Die Erschließung von MetaDaten soll sich lediglich auf relevante Bildannotationen aus der Forschungsliteratur oder auf hilfreiche Querverweise und Literaturangaben beschränken können. Eine solche Aufbereitung bietet nicht nur Experten ein mächtiges Arbeitsinstrument, sondern garantiert auch für fachfremde Forscher oder interessierte Laien einen intuitiven Zugang $\mathrm{zu}$ wertvoller Information.

Die Hauptschwierigkeit der bildinhaltlichen Recherche in digitalen Bibliotheken lassen sich in verschiedene Teile aufgliedern:

- Für jedes Anwendungsgebiet muss die spezifische Auswahl der bildinhaltlichen Features getroffen und ihre Gewichtung zueinander entsprechend der fachlichen Aussagekraft optimiert werden. Je nach Art der Anwendung können dazu sogar diverse Arten der Extraktion grafischer Information notwendig sein.

- Formbasierte Features zeigen auf dem Gebiet der Bildanalyse große Schwächen und verlangen einen kostenintensiven Vorverarbeitungsschritt, in welchem relevante Formen auf jedem Bild ausgezeichnet werden müssen (Segmentierung). Eine Automatisierung dieses Schritts ist aus Kostengründen unumgänglich.

- Für die Kombination bewerteter Ergebnislisten miteinander müssen sowohl für homogene, als auch für heterogene Datenquellen und -strukturen effiziente Algorithmen bereitgestellt werden.

- Bei der Auslieferung von Bildmaterial muss den Anforderungen der Benutzer Rechnung getragen werden. Verschiedene Bildformate und Qualitätsstufen sollen zur Verfügung stehen, aber es darf nur ein Mindestmaß sowohl an Speicherplatz als auch an Konvertierungszeiten benötigt werden. Hier ist ein optimaler Ausgleich zu schaffen.

Im HERON-Projekt wurde versucht, Lösungen zu diesen Schwerpunkten zu finden. Dabei wurde der Wert darauf gelegt, die Algorithmen eines kommerziellen bildinhaltlichen Recherchesystems dem kunsthistorischen Anwendungsgebiet der Heraldik anzupassen, dabei aber die Möglichkeit für Erweiterungen und die Einbindung verschiedener Recherchekomponenten und heterogener Datenquellen offen zu halten. Für das spezielle Gebiet der Heraldik wurden Erfolg versprechende Segmentierungsverfahren entwickelt [12]. Eine fachlich fundierte ErschlieBung und Digitalisierung eines heraldischen Standardwerkes im Rahmen des Projekts schafft eine geeignete Grundlage zur computergestützten Arbeit im historischen und kunsthistorischen Bereich [11].
In der Systemarchitektur wurde eine MiddlewareSchicht eingesetzt, wobei effiziente Algorithmen sowohl zur Kombination bewerteter Ergebnislisten miteinander [5], als auch zur Formatoptimierung und Auslieferung multimedialer Dokumente in diversen Formaten und Qualitätsstufen benutzt werden können [13].

Weitere Fragestellungen und offene Probleme im interdisziplinären HERON-Projekt sind sowohl geisteswissenschaftlicher als auch informatischer Natur. Im kunsthistorischen Anteil des Projekts muss - neben der Fortführung der fachlich fundierten Erschließung und Digitalisierung geisteswissenschaftlicher Bestände - eine Konzentration vor allem auf der Ausweitung auf andere Anwendungsgebiete liegen. Die Schwierigkeit ist hierbei insbesondere in der Erschließung der anwendungsspezifischen Semantik zu sehen, ohne welche keine effektive bildinhaltliche Suche oder effiziente Vorverarbeitung und Segmentierung möglich sein kann.

\section{Danksagung}

Das HERON-Projekt wird im Rahmen des V3D2-Schwerpunktprogramms der Deutschen Forschungsgemeinschaft (DFG) gefördert. Die angestrebten Patente werden durch die DFG-Ideenwerkstatt unterstützt.

Die verwendete Datenbank Software DB2 und die DB2 Relational Extender wurden von IBM im Rahmen des Programms „DB2 for Educational Purposes“ zur Verfügung ge-
stellt.

\section{Literatur}

[1] Bach, Fuller, Gupta, Hampapur, Horowitz, Humphrey, Jain, Shu: Virage Image Search Engine: An Open Framework for Image Management. In: Storage and $\mathrm{Re}$ trieval for Image and Video Databases (SPIE) 1996 , pp. 76-87, 1996.

[2] Faloutsos, Barber, Flickner, Hafner, Niblack, Petkovic, Equitz: Efficient and Effective Querying by Image Content, Journal of Intelligent Information Systems, Vol. 3 (1994), pp. 231-262.

[3] Furht, Smoliar, Zhang: Video and Image Processing in Multimedia Systems, Kluwer Academic Publishers
1995 .

[4] Fagin: Combining Fuzzy Information from Multiple Systems. In: 15th ACM Symposium on Priciples of Database Systems, pp. 216-226. ACM 1996.

[5] Güntzer, Balke, Kießling: Optimizing Multi-Feature Queries for Image Databases. Proceed. of the Conference on Very Large Databases (VLDB), Kairo, 2000.

[6] Gong: Intelligent Image Databases. Kluwer Academic Publishers, 1998.

[7] Kießling, Erber-Urch, Balke, Birke, Wagner: The HERON Project - Multimedia Database Support for History and Human Sciences. Published in Dassow, Kruse (edit.). Informatik '98: Informatik zwischen Bild und Sprache, pp. 309-318, Springer Heidelberg 1998.

[8] Sheth, Shah Parasuraman, Mudumbai: Searching Distributed and Heterogeneous Digital Media: The VisualHarness approach. In: 8th Working Conference on Database Semantics - Semantic Issues in Multimedia Systems, pp 311-330. 1999.

[9] Sheth, Klas (Edit.): Multimedia Data Management: Using Metadata to Integrate and Apply Digital Media. McGraw-Hill 1998. 
[10] Subrahmanian: Priciples of Multimedia Database Systems. Morgan Kaufmann Publishers 1998.

[11] Balke, W.-T., Urch, K.: Digitalisierungspraxis im DFGProjekt HERON (Heraldry Online). Bibliotheksforum Bayern, 27, 2000.

[12] Vogel, Balke, Kießling: (Semi-)Automatic Segmentation in Historic Collections of Heraldic Images. In Proceedings of the 15th International Conference on Pattern
Recognition, ICPR 2000, Barcelona, Spain, September 2000.

[13] Wagner, Holland, Kießling: Efficient and Flexible Web Access to Art-Historical Image Collections. Proceedings of the 15th ACM Symposium on Applied Computing, SAC 2000, volume 2, pages 915-921, Como Italy, March 2000.
Prof. Dr. Werner Kießling ist Ordinarius für Praktische Informatik und Inhaber des Lehrstuhls für Datenbanken und Informationssysteme am Institut für Informatik der Universität Augsburg.

Katharina Urch m. a. Fachreferentin für Kunstgeschichte, Archäologie, Neuere Deutsche Literaturgeschichte u. Allgemeine und Vergleichende Literaturgeschichte an der Universitätsbibliothek Augsburg.
Dipl.-Math. Wolf-Tilo Balke, Dipl.-Math. Thomas Birke, Dipl.-Inf. Matthias Wagner sind wissenschaftliche Mitarbeiter am Lehrstuhl für Datenbanken und Informationssysteme (Prof. KieBling) der Universität Augsburg.

Adresse: Institut für Informatik, Universität Augsburg, D-86135 Augsburg,

E-Mail:\{kiessling,balke,birke,wagner\}@informatik.uni-augsburg.de, katharina.urch@bibliothek.uni-augsburg.de 